\title{
Activation of sickle red blood cell adhesion via integrin-associated protein/CD47-induced signal transduction
}

\author{
Julia E. Brittain, ${ }^{1}$ Kathryn J. Mlinar, ${ }^{2}$ Christopher S. Anderson, ${ }^{1}$ Eugene P. Orringer, ${ }^{3}$ \\ and Leslie V. Parise ${ }^{1}$
}

${ }^{1}$ Department of Pharmacology,

${ }^{2}$ School of Medicine, and

${ }^{3}$ Department of Medicine, The University of North Carolina at Chapel Hill, Chapel Hill, North Carolina, USA

Address correspondence to: Leslie V. Parise, Department of Pharmacology, The University of North Carolina at Chapel Hill, CB\#7365, Chapel Hill, North Carolina 27599-7365, USA.

Phone: (919) 966-2238; Fax: (919) 966-5640; E-mail: parise@med.unc.edu.

Received for publication July 20, 2000, and accepted in revised form May 9, 2001.

Peripheral human red blood cells (RBCs) are not generally known to become activated and adhesive in response to cell signaling. We show, however, that soluble thrombospondin via integrin-associated protein (IAP; CD47) increases the adhesiveness of sickle RBCs (SS RBCs) by activating signal transduction in the SS RBC. This stimulated adhesion requires occupancy of IAP and shear stress and is mediated by the activation of large $\mathrm{G}$ proteins and tyrosine kinases. Reticulocyte-enriched RBCs derived from sickle-cell disease (SCD) patients are most responsive to IAP-induced activation. These studies therefore establish peripheral SS RBCs as signaling cells that respond to a novel synergy between IAP-induced signal transduction and shear stress, suggesting new therapeutic targets in SCD.

J. Clin. Invest. 107:1555-1562 (2001).

\section{Introduction}

Chronically impaired blood flow in sickle-cell disease (SCD) leads to degenerative organ damage, whereas acute vaso-occlusive crises in SCD patients can progress to stroke, embolism, myocardial infarction, and even death (1). Recent studies have demonstrated that sickle red blood cells (SS RBCs), but not normal RBCs, can bind to vascular endothelial cells $(2,3)$, platelets (4), and the vascular adhesive proteins thrombospondin (TSP) and laminin (5-9) in the extracellular matrix. SS RBC adhesion to these vascular components is thought to promote, if not initiate, vaso-occlusion $(10,11)$.

In the circulation, TSP is normally sequestered in platelet $\alpha$-granules. Upon activation, platelets release TSP, causing plasma concentrations to increase by three to four orders of magnitude (12). TSP also exists as an integral component of the subendothelial matrix $(13,14)$. In this immobilized form, TSP is a primary adhesive substrate for endothelial cells (15) and may become exposed to flowing blood as a result of the extensive vascular damage present in SCD (16).

In SCD, both soluble and matrix TSP are proposed to play a role in blood vessel occlusion by SS RBCs and ensuing vaso-occlusive crises. We and others have demonstrated that both purified and cell-secreted TSP, when immobilized, is a primary adhesive substrate for SS RBCs under relevant flow conditions $(5,17,18,19)$. Soluble TSP, which is significantly elevated in the plasma of SCD patients (20), may also serve as a linker molecule between SS RBCs and endothelial cells $(7,8)$.
On several hematopoietic cell types, integrin-associated protein (IAP; CD47) has been shown to function as both a binding site for TSP as well as an agonist receptor in response to soluble TSP. IAP, which binds to the cellbinding domain of TSP (21), activates platelets (22), mature $T$ cells (23), and neutrophils (24). On platelets, IAP increases platelet adhesion via a $\mathrm{G}_{\mathrm{i}}$-linked signal transduction pathway $(22,25)$. IAP is expressed on normal and SS RBCs (26) and has been demonstrated to protect normal RBCs from immune clearance (27). Moreover, we previously demonstrated that under basal flow conditions, IAP is a primary cell-surface adhesive molecule on SS RBCs for immobilized TSP (26).

Although other hematopoietic cells become adhesive in response to agonists or other stimuli, SS RBCs themselves are generally regarded to adhere passively to adhesive sites in the vasculature. However, RBCs express a number of agonist receptors including purinergic receptors (28), a platelet-activating factor receptor (29), receptors for IL-8 (30, 31), GPIV or CD36 (2, 32), and IAP (26). While passive, basal SS RBC adhesion probably occurs in SCD, the expression of agonist receptors on SS RBCs suggests that SS RBC adhesion might also be regulated. Given the elevated plasma levels of TSP in SCD patients (20) and the signaling potential of these cells, we investigated a role for IAP in SS RBC activation. We demonstrate that occupancy of IAP on the SS RBC activates a shear stress-dependent, large $G$ protein-mediated signal transduction pathway that substantially promotes adhesion of SS RBCs to immobilized TSP. We also provide evidence that one or more piceatannol-sensitive tyrosine 
kinases becomes activated via a novel synergy between IAP signaling and shear-stress, suggesting that tyrosine kinase activation in the SS RBC is required for IAP-stimulated adhesion under flow conditions. These results therefore identify the SS RBC as a signal-transducing cell and document a new paradigm for the regulation of SS $\mathrm{RBC}$ adhesion in the vasculature.

\section{Methods}

Reagents. Human TSP-1 purified from platelets was obtained from Life Technologies Inc. (Grand Island, New York, USA), Calbiochem-Novabiochem Corp. (La Jolla, California, USA), Sigma Chemical Co. (St. Louis, Missouri, USA), or Cortex Biochemical (San Leandro, California, USA), and also kindly provided by Dean Mosher (University of Wisconsin at Madison, Madison, Wisconsin, USA) and Jack Lawler (Brigham and Women's Hospital and Harvard Medical School, both of Boston, Massachusetts, USA). Antibodies against IAP, B6H12, and1F7, were generous gifts from Eric Brown (University of CaliforniaSan Francisco, San Francisco, California, USA). The TGF- $\beta$ neutralizing $m A b$ was purchased from Chemicon International (Temecula, California, USA). Recombinant TGF- $\beta$ was purchased from Serotec Inc. (Raleigh, North Carolina, USA). Initially, TSP peptides 4N1K (kRFYVVMWKk) and control 4NGG (kRFYGGMWKk) were provided by William Frazier (Washington University, St. Louis, Missouri, USA) (21). These peptides were also synthesized and HPLCpurified by the University of North Carolina-Chapel Hill Protein Chemistry Laboratory. Pertussis toxin, piceatannol, and genistein were obtained from Calbiochem-Novabiochem Corp.

$R B C$ preparation. SS RBCs were obtained from homozygous SCD patients with informed consent during clinic visits to the University of North Carolina Comprehensive Sickle Cell Center or obtained from healthy controls. All blood samples were drawn by venipuncture into $0.13 \mathrm{M}$ sodium citrate and processed immediately by centrifugation at $150 \mathrm{~g}$ for 15 minutes at ambient temperature to isolate blood cells from plasma and platelets. RBCs were then washed three times in CGS $(1.29 \mathrm{mM}$ sodium citrate, $3.33 \mathrm{mM}$ glucose, and $124 \mathrm{mM} \mathrm{NaCl}[\mathrm{pH}=7.2])$. The cells were resuspended in PBS and packed at $400 \mathrm{~g}$ for 10 minutes. A $1 \%$ hematocrit was then prepared by diluting $30 \mu \mathrm{l}$ of packed RBCs per $1.5 \mathrm{ml}$ of perfusion media (HBSS supplemented with $0.3 \%$ BSA and 20 mM HEPES [ $\mathrm{pH}=7.4]$ ). This preparation essentially removed all platelets from the washed RBCs (platelet count $<250$ per $3 \times 10^{8} \mathrm{RBCs}$ ) and eliminated most white blood cells (WBCs), although WBC counts varied between preparations. Frequently, we were unable to detect WBCs in our samples by direct examination of stained preparations. On average, less than $0.5 \%$ of this preparation were WBCs in all experiments except one, in which $1-2 \%$ WBCs were present. To confirm that any WBC contamination did not compound our experimental results, we evaluated the contribution of WBCs at the maximal detected contaminating numbers (the 1-2\% range) unless otherwise noted.

WBC preparation. WBCs were prepared by layering diluted buffy coats from separated whole blood over a 1.0770-1.0800 Ficoll-sodium diatrizoate solution. The layers were then spun at $400 \mathrm{~g}$ for 25 minutes. WBCs were collected, washed in PBS, and resuspended at appropriate concentrations in perfusion media.

Flow adhesion assay and protein immobilization. The flow adhesion system used for this study was designed to mimic blood flow through postcapillary venules, as described previously (5). Briefly, purified TSP $(1.5 \mu \mathrm{g})$ in perfusion media was immobilized onto identical wells formed by a silicon gasket in a $35-\mathrm{mm}$ polystyrene culture dish by incubating for 2 hours at $37^{\circ} \mathrm{C}$. A $1 \%$ hematocrit solution $(1.5 \mathrm{ml})$ was flowed over the wells at a rate of $1.0 \mathrm{ml} / \mathrm{min}$ and constant shear stress of 1 dyne $/ \mathrm{cm}^{2}$. After a wash period of approximately 4 minutes, the number of adherent cells from four representative areas of the well was counted by direct light microscopy. The counted cells were then averaged and presented as adherent cells per square millimeter. Any occasional adherent WBC was not included in the analysis.

$S S R B C$ static adhesion assay. The static adhesion assay used for this study is based on an ATP monitoring system, ATP-Lite (Packard, Groningen, The Netherlands). Microtiter plates (Becton Dickinson, Franklin Lakes, New Jersey, USA) were coated with $1.5 \mu \mathrm{g}$ TSP for 2 hours at $37^{\circ} \mathrm{C}$ then blocked with $0.3 \%$ BSA for 30 minutes at $37^{\circ} \mathrm{C}$. SS RBCs $\left(3 \times 10^{6}\right)$ or WBCs $(6 \times$ $10^{4}$ ) were added to each well and incubated for 2 hours at $37^{\circ} \mathrm{C}$. Adherent cells were washed four times, and remaining SS RBCs or WBCs were treated with ATP-Lite for luminescence measurement in a microtiter plate reader (TopCount; Packard Instrument Co., Meriden, Connecticut, USA). Under these conditions no platelet adhesion was observed. All results were verified by direct counting of the SS RBCs (positively identified by Wright/Giemsa staining) by light microscopy (see Figure 3a) with the minimal contaminating WBC ATP subtracted out.

$R B C$ lysis and Western blotting. Cells were lysed for 20 minutes in ice-cold lysis buffer containing $1 \%$ Triton X-100, $50 \mathrm{mM}$ HEPES ( $\mathrm{pH}$ 7.4), $137 \mathrm{mM} \mathrm{NaCl}, 500$ $\mu \mathrm{M}$ EGTA, $100 \mathrm{mM} \mathrm{NaVO}_{3}, 100 \mathrm{mM} \mathrm{NaF}$, and $1 \mathrm{X}$ Protease Inhibitor Cocktail Set III (Calbiochem-Novabiochem Corp.). The Triton X-100 soluble fraction was incubated overnight at $4{ }^{\circ} \mathrm{C}$ with either the antiphosphotyrosine antibody PY20 or PY99 (Santa Cruz Biotechnology Inc., Santa Cruz, California, USA) at 2 $\mu \mathrm{g} / \mathrm{ml}$ final concentration followed by incubation (1 hour) with protein G Sepharose beads. Immunoprecipitated proteins were washed three times in lysis buffer, reduced according to the method of Laemmli (33), loaded on $4-20 \%$ SDS-PAGE, transferred to PVDF membrane (Millipore), and blotted with 4G10 anti-phosphotyrosine mAb (Transduction Laboratories, Lexington, Kentucky, USA). 

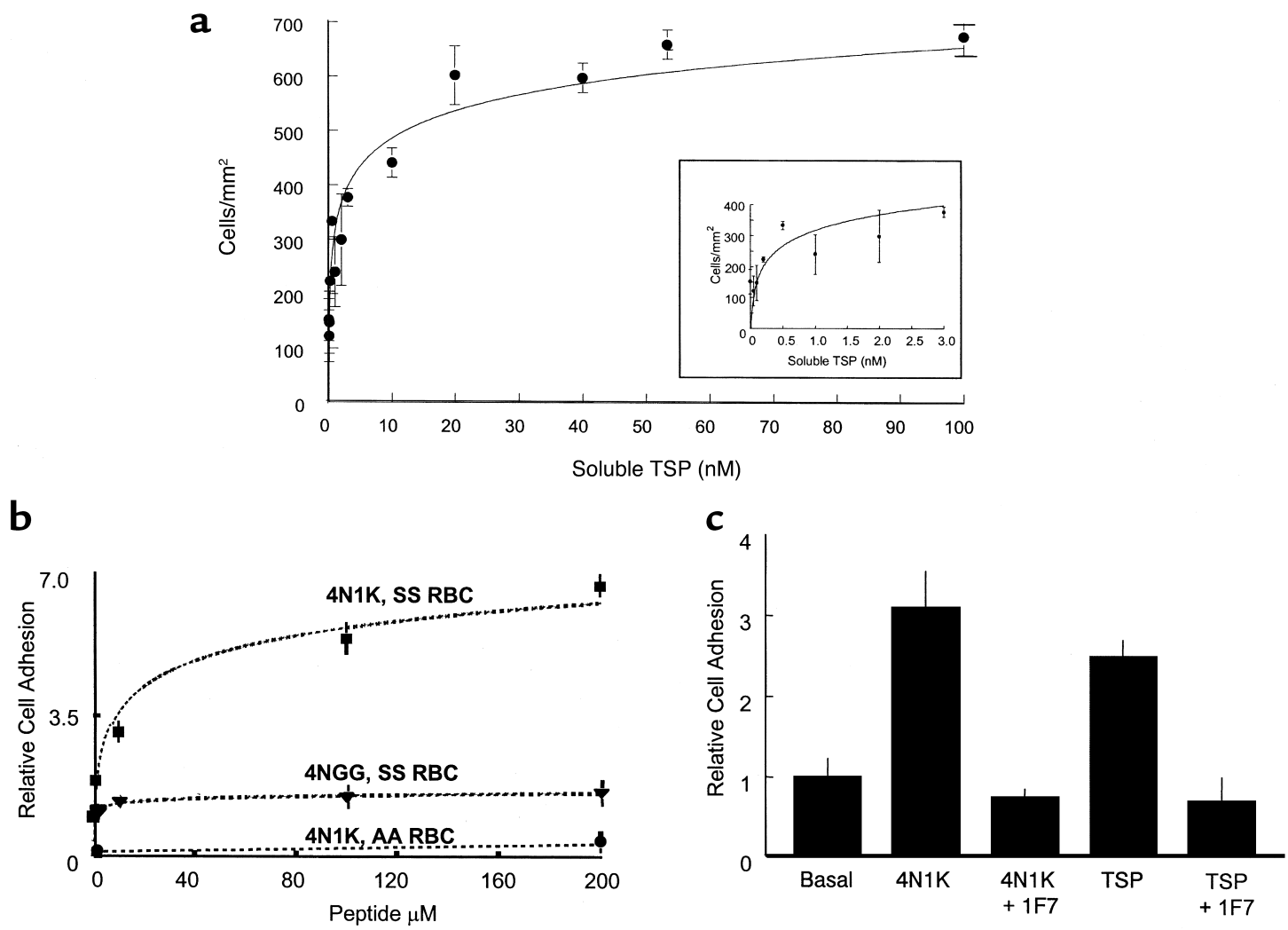

Figure 1

Soluble TSP or 4N1K agonist peptide promotes SS RBC adhesion to immobilized TSP via IAP. (a) Increasing concentrations of soluble TSP, including those reported in plasma of SCD patients (20) (inset), cause increased SS RBC adhesion to immobilized TSP under flow (flow rate: $1 \mathrm{ml} / \mathrm{min}$, shear: 1 dyne $/ \mathrm{cm}^{2}$ ). Data plotted represent results from six different patients \pm SD. (b) Increasing concentrations of $4 \mathrm{~N} 1 \mathrm{~K}$ agonist peptide ( $k R F Y W M W K k$ ) promote SS RBC adhesion to immobilized TSP (squares), whereas the 4NGG control peptide (kRFYGGMWKk) has no effect on SS RBC adhesion to immobilized TSP under flow (triangles). Data were normalized from six independent patient samples and are shown as \pm SD. $4 \mathrm{~N} 1 \mathrm{~K}$ treatment has no effect on AA RBC adhesion (circles, $n=3$ donors). (c) $4 \mathrm{~N} 1 \mathrm{~K}(16 \mu \mathrm{M})$ and TSP-stimulated $(50 \mathrm{nM})$ adhesion to immobilized TSP can be blocked by a 20-minute pretreatment of SS RBCs with $1 \mathrm{F7}(1 \mu \mathrm{g} / \mathrm{ml})$ before agonist treatment (mean of $n=3$ patients, \pm SD).

Statistics. The significance of any differences for all treatments studied was determined using a two-tailed Student's $t$ test. Unless otherwise noted, $P<0.001$.

\section{Results}

To evaluate the potential of SS RBCs to become more adhesive in response to agonist-induced signaling, we evaluated the ability of a known ligand and agonist for IAP, soluble TSP, to promote SS RBC adhesion under relevant flow conditions. Freshly drawn, washed human SS RBCs were preincubated $\left(30\right.$ minutes at $\left.37^{\circ} \mathrm{C}\right)$ with soluble TSP at concentrations reported to be present in the plasma of SCD patients (20) (Figure 1a, inset) and flowed over immobilized TSP under conditions that mimics shear stress and flow in postcapillary venules (5). Soluble TSP increased SS RBC adhesion to immobilized TSP up to sixfold that of basal SS RBC adhesion observed in the absence of soluble TSP (Figure 1a). This activation of adhesion cannot be contributed to contaminating TGF- $\beta$ that copurifies with TSP; we still observed increased SS RBC adhesion in response to soluble TSP that was preincubated with maximal concentrations of a neutralizing TGF- $\beta \mathrm{mAb}$ (data not shown). Furthermore, purified, recombinant TGF- $\beta$ had no effect on SS RBC adhesion to TSP under flow conditions (data not shown). These results indicate a potentiating effect of soluble TSP on SS RBC adhesion to immobilized TSP. Because of this promotion, rather than inhibition, of SS RBC adhesion to immobilized TSP by soluble TSP, it appears that soluble TSP binds to SS RBCs by a mechanism distinct from that of immobilized TSP, and potentially activates SS RBCs.

Because IAP is an agonist receptor in response to TSP on other cells, we evaluated its role as an agonist receptor for soluble TSP on SS RBCs with the IAP-function blocking mAb 1F7 (34). We found that TSP-stimulated adhesion was completely blocked by $1 \mathrm{~F} 7(1 \mu \mathrm{g} / \mathrm{ml})$ (Figure 1c), but not by an isotype-matched control antibody (data not shown). These results suggest that IAP is essential for the response to soluble TSP under these conditions.

To confirm that IAP occupancy can promote SS RBC adhesion to immobilized TSP, we preincubated SS RBCs with an IAP-specific agonist peptide derived from TSP, termed $4 \mathrm{~N} 1 \mathrm{~K}(21,35)$. We had previously determined that this peptide could support SS RBC adhesion under flow conditions and that IAP under basal flow conditions is an adhesion receptor on SS RBCs for TSP (26). However, under identical shear conditions as above, the 


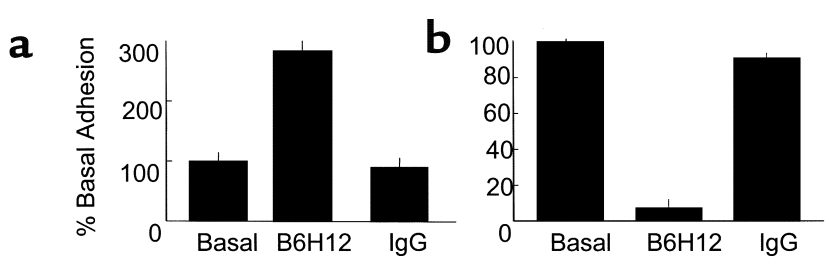

C

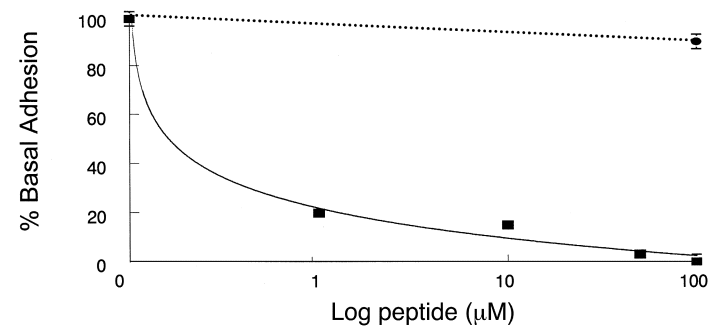

Figure 2

IAP stimulation of SS RBC adhesion is shear-dependent. (a) Preincubation (30 minutes) of SS RBCs with anti-IAP mAb B6H12 $(1 \mu \mathrm{g} / \mathrm{ml})$ increases SS RBC adhesion under flow conditions $(n=5$ patients, $\pm \mathrm{SD})$. (b) B6H12 $(1 \mu \mathrm{g} / \mathrm{ml})$ blocks SS RBC adhesion to TSP under static conditions ( $n=3$ patients). Basal, nonstimulated SS RBC adhesion is set at $100 \%$. Identical treatment with an isotypematched control antibody $(1 \mu \mathrm{g} / \mathrm{ml})$ has no effect on adhesion under flow (a) or static (b) conditions. (c) Agonist peptide 4N1K blocks SS RBC adhesion to immobilized TSP under static conditions (squares), whereas 4NGG control peptide (ovals) has little effect on static SS $\mathrm{RBC}$ adhesion ( $n=3$ patients, $\pm \mathrm{SD})$.

soluble 4N1K peptide did not block adhesion, but maximally stimulated SS RBC adhesion to immobilized TSP sevenfold (Figure 1b, squares), whereas a control peptide, 4 NGG $(21,35)$, had little effect on SS RBC adhesion (Figure 1b, triangles). This stimulatory effect of the $4 \mathrm{~N} 1 \mathrm{~K}$ peptide on adhesion was also blocked by mAb 1F7 $(1 \mu \mathrm{g} / \mathrm{ml})$, thus indicating an IAP-specific interaction with the agonist peptide (Figure 1c). This result suggests that, in this context, IAP functions as an agonist, rather than adhesion receptor, on SS RBCs. SS RBC adhesion to immobilized TSP doubled after 2 minutes and was maximal within 30 minutes of pretreatment with the $4 \mathrm{~N} 1 \mathrm{~K}$ peptide (data not shown), suggesting a relatively slow activation of adhesion. However, in contrast to SS RBCs, treatment of AA RBCs with the $4 \mathrm{~N} 1 \mathrm{~K}$ agonist peptide did not stimulate adhesion to immobilized TSP (Figure 1b, circles), suggesting that this adhesive phenotype may not be relevant to normal peripheral RBCs.

We next investigated the effects of another IAP-specific $\mathrm{mAb}, \mathrm{B} 6 \mathrm{H} 12$, on SS RBC adhesion to immobilized TSP. B6H12 blocks ligand binding to IAP on most cells $(22,34)$, but stimulates IAP signaling in at least one cell type (23). We observed that B6H12 increased SS RBC adhesion to immobilized TSP under shear conditions (Figure 2a). This result further supports a role for IAP as an agonist receptor on SS RBCs that activates adhesion.

To determine the contribution of shear stress to TSP agonist-induced adhesion, we examined SS RBC adhesion to immobilized TSP in the absence of shear. For this purpose, a static adhesion assay was designed in a 96-well format. We found two immediate differences between sickle cell adhesion under shear versus static conditions. First, although B6H12 stimulated SS RBC adhesion in the flow adhesion assay (Figure 2a), treatment with this $\mathrm{mAb}$, but not an isotype-matched control mAb, completely blocked SS RBC adhesion to TSP in the static adhesion assay (Figure 2b). Second, although the $4 \mathrm{~N} 1 \mathrm{~K}$ agonist peptide stimulated sickle cell adhesion to immobilized TSP under flow (Figure $1 \mathrm{~b})$, SS RBC treatment with the $4 \mathrm{~N} 1 \mathrm{~K}$ peptide (squares), but not the control 4NGG peptide (ovals), completely inhibited sickle cell adhesion to immobilized TSP in the static assay (Figure 2c). These results suggest that under static conditions, IAP is a major sickle cell adhesion receptor for immobilized TSP. These data also suggest that shear stress provides an essential component to the activation of SS RBC adhesion that occurs in response to $4 \mathrm{~N} 1 \mathrm{~K}$, soluble TSP or B6H12 binding to IAP on the SS RBC. Thus, although IAP clearly appears to be a major static adhesion receptor for immobilized TSP, our results also suggest that IAP can function as an agonist receptor that, under relevant flow conditions, potentially activates sheardependent signal transduction in the SS RBCs.

We next tested our hypothesis that IAP activates a signaling pathway in SS RBCs that, under flow conditions, promotes adhesion to immobilized TSP. In

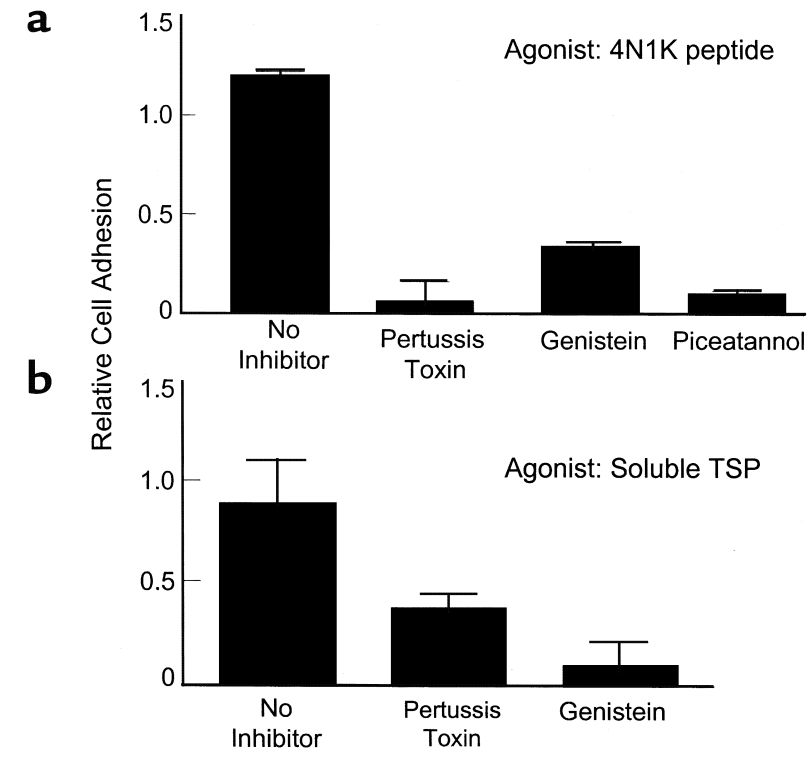

\section{Figure 3}

IAP-induced adhesion of SS RBCs requires $G_{i}$ and tyrosine kinase activity. (a) SS RBC adhesion to immobilized TSP under flow conditions, stimulated by a submaximal concentration of agonist peptide $4 \mathrm{~N} 1 \mathrm{~K}$ $(16 \mu \mathrm{M})$, is completely blocked by pretreatment (30 minutes) with the large G protein inhibitor pertussis toxin $(1 \mu \mathrm{g} / \mathrm{ml})$ or the tyrosine kinase inhibitors genistein $(260 \mu \mathrm{M})$ or piceatannol $(30 \mu \mathrm{g} / \mathrm{ml})$. (b) SS RBC adhesion to immobilized TSP that is stimulated by a submaximal concentration of soluble TSP $(2 \mathrm{nM})$ is partially blocked by preincubation with pertussis toxin $(1 \mu \mathrm{g} / \mathrm{ml})$ and completely blocked with genistein. Normalized data with basal adhesion subtracted out represent four $4 \mathrm{~N} 1 \mathrm{~K}$ - and two TSP-treated patient samples, respectively $( \pm \mathrm{SD})$. 
platelets and smooth muscle cells, IAP functionally couples to, and signals via, the large $G$ protein $G_{i}(22$, 36). In addition, several nonreceptor tyrosine kinases have been implicated in IAP signaling $(22,23)$. To examine potential IAP signaling in SS RBCs, we pretreated SS RBCs with the $\mathrm{G}_{\mathrm{i}}$ inhibitor pertussis toxin (1 $\mu \mathrm{g} / \mathrm{ml}, 30$ minutes) and stimulated the cells with the $4 \mathrm{~N} 1 \mathrm{~K}$ agonist peptide. Pertussis toxin treatment almost completely blocked the 4N1K-stimulated component of SS RBC adhesion to immobilized TSP (Figure 3a), but had no effect on basal, nonagonist-stimulated adhesion to TSP (data not shown), suggesting a role for $G_{i}$ activation downstream of IAP in SS RBCs. Pertussis toxin also blocked soluble TSP-stimulated adhesion to immobilized TSP, but by about 50\% (Figure $3 \mathrm{~b}$ ), suggesting that soluble TSP may act via multiple signaling pathways in the SS RBCs (Figure 3b). Consistent with a role for tyrosine kinase activity in IAP signaling, the tyrosine kinase inhibitor piceatannol (30 $\mu \mathrm{g} / \mathrm{ml}$ ) also blocked $4 \mathrm{~N} 1 \mathrm{~K}$-stimulated SS RBC adhesion to immobilized TSP under flow conditions. Treatment with genistein $(260 \mu \mathrm{M})$, another tyrosine kinase inhibitor, also partially blocked the SS RBC response to the $4 \mathrm{~N} 1 \mathrm{~K}$ peptide (Figure $3 \mathrm{a}$ ), but completely blocked the response to soluble TSP (Figure $3 \mathrm{~b}$ ), further implying that tyrosine kinase become activated as a result of $4 \mathrm{~N} 1 \mathrm{~K}$ or TSP stimulation. Neither inhibitor had any effect on basal SS RBC adhesion (data not shown). These results indicate that $4 \mathrm{~N} 1 \mathrm{~K}$-induced IAP signaling in SS RBCs is similar to that in other agonistregulated cells and involves the activation of large $G$ proteins and tyrosine kinases.

Another potent inducer of intracellular signaling in several cell types is the shear stress that results from exposure of cells to blood flow. Endothelial cells exposed to shear exhibit marked differences in receptor expression, differentiation, and cell signaling $(37,38)$. Likewise, platelets exposed to high shear arterial blood flow can spontaneously activate (39) and adhere to the blood vessel wall by mechanisms distinct from platelets under slower venous flow conditions $(40,41)$.

To explore further the shear-induced component of IAP signaling in SS RBCs, we exposed nonstimulated SS RBCs to a 2-minute pulse of shear identical to that present in the flow adhesion assay. The cells were lysed immediately after shear and were analyzed by immunoprecipitation of tyrosine-phosphorylated proteins followed by SDS-PAGE and immunoblotting. As shown in a representative blot (Figure 4a), exposure of SS RBCs to shear alone induced both the phosphorylation and dephosphorylation of several protein bands relative to SS RBCs under static conditions. Most notably, proteins migrating at approximately 100 and $70 \mathrm{kDa}$ became markedly phosphorylated, whereas proteins migrating at approximately 45 and $27 \mathrm{kDa}$ lost phosphotyrosine content. These results show that both shear-dependent tyrosine phosphorylation and dephosphorylation of proteins occur in the SS RBCs and further support a role for shear stress in the activation of
SS RBC adhesion. To investigate the combined effects of IAP-mediated signaling and shear on cellular protein phospho-tyrosine content, we stimulated SS RBCs with the $4 \mathrm{~N} 1 \mathrm{~K}$ agonist peptide $(100 \mu \mathrm{M})$, exposed the cells to shear stress, and analyzed phosphotyrosine content as above. The combination of agonist peptide and shear stress further increased the tyrosine phosphorylation of the 100- and 70-kDa bands and caused the appearance of several additional bands (Figure 4b, lane 3 ). This increase in tyrosine phosphorylation of the 100- and 70$\mathrm{kDa}$ bands was completely blocked by pretreatment of SS RBCs with piceatannol (Figure 4c, lane 2). Examination of the phosphotyrosine content of an overestimate of contaminating numbers of purified WBCs $(200,000$ cells) or platelets $(50,000)$ under identical treatment conditions indicated a lack of detectable tyrosine phosphorylation in these cells, even under shear stress coupled with $4 \mathrm{~N} 1 \mathrm{~K}$ stimulation (Figure 4b, lanes 1 and 2).

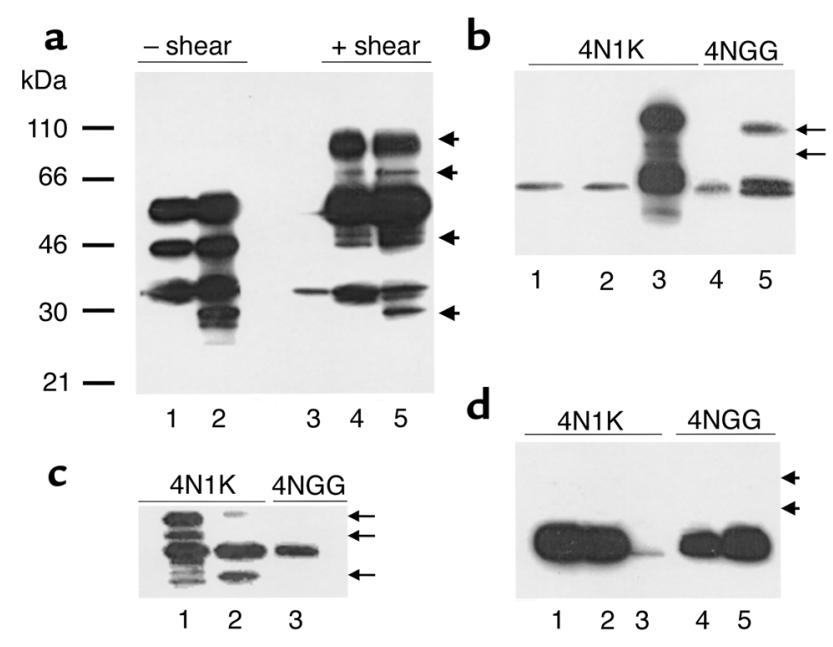

\section{Figure 4}

Shear stress and IAP stimulation induce tyrosine phosphorylation and dephosphorylation in SS RBCs. (a) SS RBCs $\left(1 \times 10^{7}\right)$ were lysed after 2 minutes of shear stress alone $\left(1 \mathrm{dyne} / \mathrm{cm}^{2}\right)$, and tyrosine phosphorylated proteins were immunoprecipitated with PY20 (lanes 2 and 5), PY99 (lanes 1 and 4), or control IgG (lane 3) and Western blotted with 4G10. Exposure to shear induces the phosphorylation of several bands at $\sim 100 \mathrm{kDa}$ and $70 \mathrm{kDa}$ (arrows). Notable dephosphorylation occurs at approximately 45, 30, and $27 \mathrm{kDa}$ (representative of three patients). (b) $4 \mathrm{~N} 1 \mathrm{~K}(100 \mu \mathrm{M})$ plus shear induces the further phosphorylation at $100 \mathrm{kDa}$ and $70 \mathrm{kDa}$ relative to $4 \mathrm{NGG}$ plus shear (lane 5). Control IgG fails to detect this phosphorylation (lane 4). NeitherWBCs $(200,000$, lane 1$)$ nor platelets $(50,000$, lane 2) exhibit any change in phosphorylation (blot representative of four patients). (c) Pretreatment with piceatannol (lane 2) blocks tyrosine phosphorylation of the 100- and 70-kDa bands induced by shear plus $4 \mathrm{~N} 1 \mathrm{~K}$ treatment (lane 1, top arrows) back to control peptide levels (lane 3 ) (blot representative of two patients). Piceatannol potentiated the $4 \mathrm{~N} 1 \mathrm{~K}$-induced phosphorylation of a $33-\mathrm{kDa}$ band (lane 2, lower arrow). (d) Normal (AA) RBCs do not exhibit any change in tyrosine phosphorylation in response to $4 \mathrm{~N} 1 \mathrm{~K}$ and shear stimulation (blot representative of four donors and exposed eight times longer than SS blots) using either PY99 (lanes 1 and 4), PY20 (lanes 2 and 5), or control IgG (lane 3). Note the lack of phosphorylated bands at $100 \mathrm{kDa}$ and $70 \mathrm{kDa}$ (arrows). 


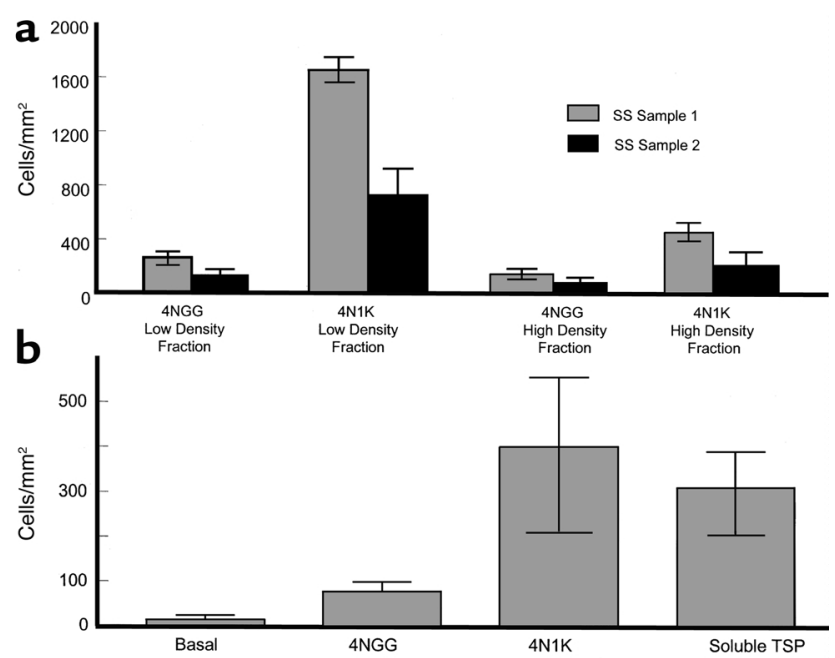

Figure 5

The low-density (reticulocyte-enriched) fraction of SS RBCs is most responsive to IAP stimulation. (a) Reticulocyte-enriched fractions of SS RBCs from two patients were prepared by centrifuging washed SS RBC over an arabinogalactan gradient (Larex Inc., White Bear Lake, New Jersey, USA) $\left(74,000 \mathrm{~g}, 20^{\circ} \mathrm{C}, 45\right.$ minutes). SS RBCs collected from low- and high-density fractions were resuspended in perfusion media and packed at $400 \mathrm{~g}$. A $1 \%$ hematocrit of each fraction was prepared, treated with or without $50 \mu \mathrm{M} 4 \mathrm{~N} 1 \mathrm{~K}$ peptide, and flowed over immobilized TSP. (b) AA reticulocytes respond to $4 \mathrm{~N} 1 \mathrm{~K}$ stimulation. Washed $\mathrm{RBCs}$ from a patient with a hemolytic anemia and increased reticulocyte count were treated with $50 \mu \mathrm{M} 4 \mathrm{~N} 1 \mathrm{~K}$ agonist, $50 \mu \mathrm{M}$ control 4NGG peptide, or $2 \mathrm{nM}$ soluble TSP and flowed over immobilized TSP. Data are presented \pm SD ( $n=2$ experiments) and are expressed in both panels as adherent cells per square millimeter.

These data indicate that the change in protein tyrosine phosphorylation from both shear stress and $4 \mathrm{~N} 1 \mathrm{~K}$ peptide stimulation occurs in the SS RBCs. Furthermore, treatment of SS RBCs with piceatannol blocked 4N1Kinduced tyrosine phosphorylation (Figure 4c, lane 2), consistent with this inhibitor's ability to block IAPstimulated adhesion. These results demonstrate that the SS RBC is initiating cell signaling in response to changes in its environment that can result in increased adhesion of these cells.

Interestingly, the $4 \mathrm{~N} 1 \mathrm{~K}$ peptide can induce moderate tyrosine phosphorylation of the $100-$ and $70-\mathrm{kDa}$ bands in the absence of shear (data not shown). Furthermore, immobilized TSP also induces tyrosine phosphorylation of these protein bands under static conditions (data not shown). Yet, the presence of either agonist alone fails to activate SS RBC adhesion in the absence of shear. These results further indicate that full IAP stimulation of SS RBC adhesion absolutely requires a novel synergy between shear stress and receptor ligation. Additionally, AA RBCs, which failed to functionally respond to agonist peptide stimulation (Figure 1b), also exhibited almost no change in amounts of tyrosine phosphorylated proteins under any of the tested conditions (Figure 4d), again suggesting an SS RBC-specific response to IAP stimulation and shear.
Given that reticulocytes may retain several signaling pathways and molecules that are lost upon normal RBC maturation (42), we hypothesized that it was these young RBCs that were mounting the signaling response to IAP. Therefore, we separated SS RBCs on an arabinogalactan gradient (26) and tested the ability of the lowdensity (reticulocyte-enriched) and high-density (reticulocyte-depleted) fractions to respond to $4 \mathrm{~N} 1 \mathrm{~K}$ stimulation under flow conditions. The low-density fraction exhibited a robust response to the $4 \mathrm{~N} 1 \mathrm{~K}$ but not the 4 NGG peptide, whereas the reticulocyte-depleted fraction was markedly deficient in response to $4 \mathrm{~N} 1 \mathrm{~K}$ stimulation of adhesion in two separate patients (Figure 5a). These results strongly suggest that the low-density, reticulocyte-enriched fraction of SS RBCs represents the most reactive population of SS RBCs. Moreover, RBCs from a patient with an idiopathic hemolytic anemia and markedly elevated reticulocyte count (35-40\%), but AA hemoglobin, clearly responded to $4 \mathrm{~N} 1 \mathrm{~K}$ agonist peptide or soluble TSP stimulation under flow (Figure $5 \mathrm{~b}$ ), suggesting that an increased reticulocyte population is sufficient to confer a response to IAP stimulation. Interestingly, this patient's RBCs, unlike SS RBCs, failed to adhere basally to immo-

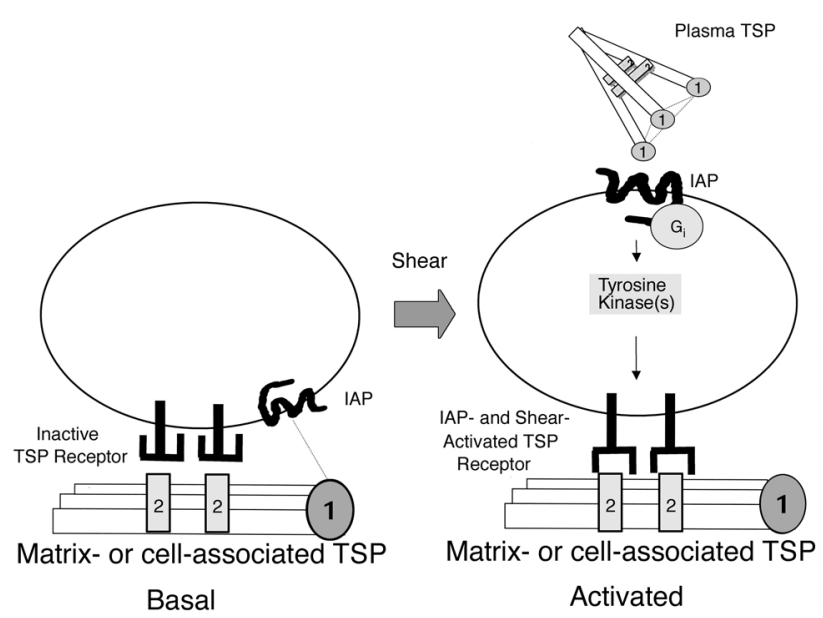

\section{Figure 6}

Model of SS RBC activation by plasma TSP and shear stress. We previously demonstrated that basal or resting SS RBCs (left) could adhere to immobilized TSP (representing subendothelial matrix- or cell-associated TSP in vivo) by an IAP-mediated adhesion to the "cell binding domain" of TSP (binding site 1) when soluble TSP levels are negligible, as in individuals who do not have SCD. In contrast, when plasma levels of TSP are elevated, as in SCD, we propose that IAP is largely occupied by plasma TSP and unable to participate directly in adhesion to immobilized TSP. However, IAP stimulation by plasma TSP in combination with physiological shear stress, via a $\mathrm{G}_{i-}$ and tyrosine kinase-dependent pathway, activates a distinct, yet unidentified, TSP receptor that preferentially recognizes immobilized matrix- or cellassociated TSP (binding site 2). We further propose that site 2 is relatively inaccessible in plasma TSP, thus explaining the marked IAPmediated activation of SS RBCs by soluble TSP, instead of an inhibition of adhesion to immobilized TSP. Finally, we demonstrate that activation of a distinct TSP receptor more than compensates for a loss of direct IAP adhesion to immobilized TSP, therefore promoting overall SS RBC avidity for immobilized TSP. 
bilized TSP under flow conditions (J.E. Brittain and L.V. Parise, unpublished observations), which might be explained by a physical difference in IAP that we previously noted on SS RBCs (26). These results further suggest that the more immature RBCs in SCD, and perhaps other anemias, respond to agonist stimulation.

\section{Discussion}

In summary, we show an unprecedented role for IAPmediated signaling in sickle erythrocytes and demonstrate a new potential pathological role for this receptor in SCD. We find that in the presence of shear stress, IAP specifically activates SS RBC adhesion to immobilized TSP via a $\mathrm{G}_{\mathrm{i}}$, and tyrosine kinase-dependent pathway. Our data suggest that this synergy between IAP and shear leads to the activation of as yet uncharacterized TSP receptors on SS RBCs that preferentially bind to immobilized, or matrix TSP, in the presence of soluble TSP or the $4 \mathrm{~N} 1 \mathrm{~K}$ agonist peptide (43).

In a previous study, we identified IAP as a TSP receptor on SS RBCs that mediates a significant amount of the basal adhesion of these cells to immobilized platelet TSPs under relevant flow conditions (26). As illustrated in a model (Figure 6), our data also suggest that with elevated plasma TSP as in SCD, IAP is largely occupied by soluble TSP, which activates a distinct TSP receptor(s) that efficiently binds to an adhesive, exposed site on TSP (binding site 2) when TSP is constrained on a solid-phase support. We predict that such conformations of TSP would be presented to SS RBCs in flowing blood either as exposed matrix TSP or as plasma TSP bound to the endothelium. The dependence of TSPinduced signaling on shear stress further suggests that the resulting adhesiveness of SS RBCs may be most relevant in the initiation of vaso-occlusion in flowing blood, which would serve to compromise blood flow, induce turbulence, and slow transit through the vasculature. In contrast, under largely static conditions, which would be expected behind a vaso-occlusion, our results suggest that IAP may function largely as an adhesion receptor for TSP bound to various sites in the vasculature, ultimately propagating vaso-occlusion. Therefore, we believe that IAP is a viable, twofold therapeutic target whose disruption could decrease the frequency and duration of vaso-occlusion in SCD.

It is intriguing that while SS RBCs respond to shear and IAP signaling in SCD, normal peripheral RBCs, which express IAP and are constitutively exposed to shear in vivo, remain nonresponsive under any of our studied conditions. The rapid clearance of SS RBCs leads to an increased reticulocyte population and younger average age of all circulating RBCs in SCD patients. Our data suggest that these young peripheral cells retain signaling molecules and pathways lost in more mature RBCs and, as a result, are the most responsive to IAP-induced SS $\mathrm{RBC}$ signaling. In fact, peripheral reticulocytes are rare in normal peripheral blood, and the minimal numbers of these cells in normal donors could account for the lack of response of normal patients. Therefore, our data also sug- gest that several pathological anemias may render patients vulnerable to increased reticulocyte adhesion as a result of signal transduction in the cells.

We propose that SCD patients would be particularly vulnerable to TSP-stimulated RBC adhesion, due to both elevated plasma levels of TSP and increased reticulocyte counts $(1,20)$. Under shear conditions, soluble TSP, present presumably in multiple conformations in the plasma of SCD patients, could induce IAP-activated sickle cell adhesion to TSP bound to endothelial cells or exposed in the subendothelial matrix in vivo, dramatically promoting vaso-occlusion. In fact, a study by Kumar et al. in which $\alpha 4 \beta 1$-mediated SS RBC adhesion to endothelial cells appeared to be upregulated by IL-8 or PMA (44), is consistent with our results that signal transduction in SS RBCs can be a basis for increasing sickle cell adhesion. Our data demonstrate, however, that IAP-mediated signaling in combination with shear-stress increases adhesiveness of SS RBCs, thus providing a new paradigm of sickle cell adhesion.

Our data thus far suggest that SS RBCs may be capable of activating multiple signaling pathways, as $4 \mathrm{~N} 1 \mathrm{~K}-$ and TSP-stimulated pathways are affected in a similar but not identical manner to signaling inhibitors. SS RBCs express at least one additional TSP receptor, GPIV or CD36, a known signaling receptor on other cells that may affect the TSP- but not 4N1K-induced SS RBC signaling. Also, the points at which shear and IAP signaling impinge in SS RBCs, and the identity of the IAPactivated TSP receptor on SS RBCs, are unknown but currently under investigation. Identification of each of the signaling pathways stimulated by IAP, shear and/or soluble TSP increases the likelihood that additional therapeutic targets will be identified to downregulate SS RBC adhesion and vaso-occlusive crises in SCD.

\section{Acknowledgments}

The authors thank William Frazier for initially providing $4 \mathrm{~N} 1 \mathrm{~K}$ and $4 \mathrm{NGG}$ peptides and for helpful scientific discussion, Eric Brown and Frederik Lindberg (Washington University) for providing anti-IAP mAb's and helpful discussions, and Jack Lawler and Dean Mosher for generously providing purified TSP. This work was supported by grants from the NIH: HL58939 (L.V. Parise) and RR00046 (E.P. Orringer).

\footnotetext{
1. Wang, W.C., and Lukens, J.N. 1999. Sickle cell anemia and other sickling syndromes. In Wintrobe's clinical hematology. Volume 1. G.R. Lee et al., editors. Williams \& Wilkins. Baltimore, Maryland, USA. 1346-1398.

2. Swerlick, R., Eckman, J., Kumar, A., Jeitler, M., and Wick, T. 1993. Alpha 4 beta 1 expression on sickle reticulocytes: vascular cell adhesion molecule 1 dependent binding to the endothelium. Blood. 82:1891-1899.

3. Hebbel, R.P., 1980. Abnormal adherence of sickle erythrocytes to cultured vascular endothelium. J. Clin. Invest. 65:154-160.

4. Wun, T., et al. 1999. Platelet-erythrocyte adhesion in sickle cell disease. J. Investig. Med. 47:121-127.

5.Joneckis, C.C., Shock, D.D., Cunningham, M.L., Orringer, E.P., and Parise, L.V. 1996. Glycoprotein IV-independent adhesion of sickle red blood cells to immobilized thrombospondin under flow conditions. Blood. 87:4862-4870.

6. Lee, S.P., et al. 1998. Sickle cell adhesion to laminin: potential role for the Q5 chain. Blood. 92:2951-2958.

7. Brittain, H.A., Eckman, J.R., Swerlick, R.A., Howard, R.J., and Wick, T.M. 1993. Thrombospondin from activated platelets promotes sickle ery-
} 
throcyte adherence to human microvascular endothelium under physiologic flow: a potential role for platelet activation in sickle cell vasoocclusion. Blood. 81:2137-2143.

8. Gupta, K., Gupta, P., Solovey, A., and Hebbel, R.P. 1999. Mechanism of interaction of thrombospondin with human endothelium and inhibition of sickle erythrocyte adhesion to human endothelial cells by heparin. Biochim. Biophys. Acta. 1453:63-73.

9. Sugihara, K., Sugihara, T., Mohandas, N., and Hebbel, R.P. 1992. Thrombospondin mediates adherence of CD36+ sickle reticulocytes to endothelial cells. Blood. 80:2634-2642.

10. Harlan, J.M. 2000. Anti-adhesion therapy in sickle cell disease. Blood. 95:365-367.

11. Hebbel, R.P. 1997. Adhesive interactions of sickle erythrocytes with endothelium. J. Clin. Invest. 100(Suppl.):S83-S86.

12. Walz, D.A. 1992. Thrombospondin as a mediator of cancer cell adhesion in metastasis. Cancer Metastasis Rev. 11:313-324.

13. Mosher, D., Doyle, M., and Jaffe, E. 1982. Synthesis and secretion of thrombospondin by cultured endothelial cells. J. Cell Biol. 93:343-348.

14. Jaffe, E., et al. 1983. Cultured human fibroblasts synthesize and secrete thrombospondin and incorporate it into extracellular matrix. Proc. Natl. Acad. Sci.USA. 80:998-1002.

15. Murphy-Ullrich, J., and Mosher, D. 1987. Interactions of thrombospondin with endothelial cells: receptor mediated binding and degradation. J. Cell Biol. 105:1603-1611.

16. Solovey, A., et al. 1997. Circulating activated endothelial cells in sickle cell anemia. N. Engl. J. Med. 337:1584-1590.

17. Manodori, A.B., Matsui, N.M., Chen, J.Y., and Embury, S.H. 1998. Enhanced adherence of sickle erythrocytes to thrombin-treated endothelial cells involves interendothelial cell gap formation. Blood. 92:3445-3454.

18. Hillery, C.A., Du, M.C., Montgomery, R.R., and Scott, J.P. 1996. Increased adhesion of erythrocytes to components of the extracellular matrix: isolation and characterization of a red blood cell lipid that binds thrombospondin and laminin. Blood. 87:4879-4886.

19. Barabino, G.A., et al. 1997. Inhibition of sickle erythrocyte adhesion to immobilized thrombospondin by von Willebrand factor under dynamic flow conditions. Blood. 89:2560-2567.

20. Browne, P.V., Mosher, D.F., Steinberg, M.H., and Hebbel, R.P. 1996. Disturbance of plasma and platelet thrombospondin levels in sickle cell disease. Am. J. Hematol. 51:296-301.

21. Gao, A.G., et al. 1996. Integrin-associated protein is a receptor for the Cterminal domain of thrombospondin. J. Biol. Chem. 271:21-24.

22. Chung, J., Gao, A.G., and Frazier, W.A. 1997. Thrombspondin acts via integrin-associated protein to activate the platelet integrin alphaIIbbeta3. J. Biol. Chem. 272:14740-14746.

23. Ticchioni, M., et al. 1997. Integrin-associated protein (CD47) is a comitogenic molecule on CD3-activated human T cells. J. Immunol. 158:677-684.

24. Lindberg, F.P., et al. 1996. Decreased resistance to bacterial infection and granulocyte defects in IAP-deficient mice. Science. 274:795-798.

25. Chung, J., Wang, X.Q., Lindberg, F.P., and Frazier, W.A. 1999. Thrombospondin-1 acts via IAP/CD47 to synergize with collagen in alpha2beta1-mediated platelet activation. Blood. 94:642-648.
26. Brittain, J.E., Mlinar, K.J., Anderson, C.S., Orringer, E.P., and Parise, L.V. 2001. Integrin-associated protein is an adhesion receptor on sickle red blood cells for immobilized thrombospondin. Blood. 97:2159-2164.

27. Oldenborg, P.A., et al. 2000. Role of CD47 as a marker of self on red blood cells. Science. 288:2051-2054.

28. Di Virgilio, F., et al. 2001. Nucleotide receptors: an emerging family of regulatory molecules in blood cells. Blood. 97:587-600.

29. Joseph, R., Welch, K.M., D’Andrea, G., and Riddle, J.M. 1989. Plateletactivating factor and red blood cells. Thromb. Res. 53:629-633.

30. Darbonne, W.C., et al. 1991. Red blood cells are a sink for interleukin 8 , a leukocyte chemotaxin. J. Clin. Invest. 88:1362-1369.

31. Neote, K., Darbonne, W., Ogez, J., Horuk, R., and Schall, T. 1993. Identification of a promiscuous inflammatory peptide receptor on the surface of red blood cells. J. Biol. Chem. 268:12247-12249.

32. Joneckis, C.C., Ackley, R.L., Orringer, E.P., Wayner, E.A., and Parise, L.V. 1993. Integrin alpha 4 beta 1 and glycoprotein IV (CD36) are expressed on circulating reticulocytes in sickle cell anemia. Blood. 82:3548-3555.

33. Laemmli, U.K. 1970. Cleavage of structural proteins during the assembly of the head of bacteriophage T4. Nature. 227:680-685.

34. Rosales, C., Gresham, H.D., and Brown, E.J. 1992. Expression of the 50$\mathrm{kDa}$ integrin-associated protein on myeloid cells and erythrocytes. $J$. Immunol. 149:2759-2764.

35. Gao, A.G., Lindberg, F.P., Dimitry, J.M., Brown, E.J., and Frazier, W.A. 1996. Thrombospondin modulates alpha v beta 3 function through integrin-associated protein. J. Cell Biol. 135:533-544.

36. Wang, X.Q., Lindberg, F.P., and Frazier, W.A. 1999. Integrin-associated protein stimulates alpha2beta1-dependent chemotaxis via Gi-mediated inhibition of adenylate cyclase and extracellular-regulated kinases. J. Cell Biol. 147:389-400.

37. Faller, D.V. 1999. Endothelial cell responses to hypoxic stress. Clin. Exp. Pharmacol. Physiol. 26:74-84.

38. Malek, A.M., Alper, S.L., and Izumo, S. 1999. Hemodynamic shear stress and its role in atherosclerosis. JAMA. 282:2035-2042.

39. Kroll, M.H., Hellums, J.D., McIntire, L.V., Schafer, A.I., and Moake, J.L. 1996. Platelets and shear stress. Blood. 88:1525-1541.

40. Sugimoto, M., et al. 1999. Shear-dependent functions of the interaction between soluble von Willebrand factor and platelet glycoprotein $\mathrm{Ib}$ in mural thrombus formation on a collagen surface. Int. J. Hematol. 69:48-53.

41. Turitto, V.T., and Hall, C.L. 1998. Mechanical factors affecting hemostasis and thrombosis. Thromb. Res. 92(Suppl.):S25-S31.

42. Kesselring, F., Spicher, K., and Porzig, H. 1994. Changes in G protein pattern and in $\mathrm{G}$ protein-dependent signaling during erythropoietin- and dimethylsulfoxide-induced differentiation of murine erythroleukemia cells. Blood. 84:4088-4098.

43. Magnetto, S., et al. 1998. CD36 mediates binding of soluble thrombospondin-1 but not cell adhesion and haptotaxis on immobilized thrombospondin-1. Cell Biochem. Funct. 16:211-221.

44. Kumar, A., Eckmam, J.R., Swerlick, R.A., and Wick, T.M. 1996. Phorbol ester stimulation increases sickle erythrocyte adherence to endothelium: a novel pathway involving alpha 4 beta 1 integrin receptors on sickle reticulocytes and fibronectin. Blood. 88:4348-4358. 